\title{
The energy structure of free electrons for semiconductor in the field of cylindrical symmetry
}

\author{
J.M. Stakhira, R.J. Stakhira \\ Ivan Franko National University, 50 Dragomanova Str. 79005 Lviv, Ukraine \\ e-mail: stakhira@rd.wups.lviv.ua
}

\begin{abstract}
The energetic structure of semiconductor free electrons in the field of cylindrical symmetry whose radial part is a sum of two components, which depend on radial coordinate according to quadratic and biquadratic laws has been studied. The quantization conditions for energy in this field are found and the importance of both quadratic and biquadratic component quantum state formation is analyzed. It is shown that these states can be described by generalized degenerated hypergeometric functions. It is revealed that presence of the biquadratic component is important for the structure of energy levels.
\end{abstract}

Keywords: free electrons, biquadratic potential, nonuniform field.

Paper received 17.12.02; accepted for publication 18.03.03.

\section{Introduction}

In most cases the changes in free electron energetic structure of a semiconductor that occur in uniform outside fields can be easily analyzed. They are also commonly known. In the case of nonuniform fields, which is more real, such an analysis is complicated and can be carried out only for a specific situation.

The changes caused by the field of a cylindrical symmetry potential are studied in this work. We consider the case of two partials of this potential, one of them being quadratic and another biquadratic function of radial coordinate. The ratio of two parts of the potential has an arbitrary value. For example, outside the field connected with the potential of such a kind, there is nonuniform magnetic field [1], magnetic induction of which includes the following parts

$$
B_{x}=-b y ; \quad B_{y}=b x ; \quad B_{z}=0 .
$$

For this magnetic field, the vector potential $A(\vec{r})$ can be chosen with the following terms

$$
A_{x}=0 ; \quad A_{y}=0 ; \quad A_{z}=-A_{0}\left(x^{2}+y^{2}\right) \text {. }
$$

Such a model of potential can be useful for studying the electron states in nanowires [2], incommensurate systems [3] and other low-dimensional systems.

The electromagnetic field of low frequency excited in a semiconductor by using variable mechanical stresses is another example of the field of this kind. Particularly, this stress causing the time dependence of the internal crystalline field in semiconductors of strong anisotropy with layered structure and intercalled phases on their basis can produce electromagnetic field, vector potential of which is given by expression (2).

In the case when such a field is produced mainly by only one constituent of the field $E_{z}$, the quantity $A_{0}$ in (2) has the value

$$
A_{0}=\frac{1}{4} \varepsilon \mu \Omega E_{z},
$$

where $\varepsilon$ and $\mu$ are the dielectric and magnetic penetrabilities, $\Omega$ is the frequency of mechanical stress. Here we shall restrict our consideration by the model of such kind of fields. 


\section{Schrödinger equation for the electron in the field of cylindrical symmetry potential and its solution}

The Schrödinger equation for the free electron existing in the field of the above considered potential in the effective mass approach has the form

$$
\begin{aligned}
& {\left[-\hbar^{2}\left(\frac{\partial^{2}}{\partial x^{2}}+\frac{\partial^{2}}{\partial y^{2}}+\frac{\partial^{2}}{\partial z^{2}}\right)+\right.} \\
& +2 e A_{0}\left(x^{2}+y^{2}\left(-i \hbar \frac{\partial}{\partial z}\right)+e^{2} A_{0}^{2}\left(x^{2}+y^{2}\right)^{2}\right] \times \\
& \times \psi(x, y, z)=2 m^{*} E \psi(x, y, z) .
\end{aligned}
$$

It is favorable to change from the Cartesian to cylindrical coordinates presenting primary the wave function $\psi(x, y, z)$ in the form

$$
\psi(x, y, z)=\psi(\rho) e^{i\left(k_{z} z+m \psi\right)}
$$

where $m=0, \pm 1, \pm 2 \ldots, k_{z}$ is the wave vector component. In this case, for the function we have the following equation

$$
\begin{aligned}
& \frac{d^{2}}{d \rho^{2}} \psi(\rho)+\frac{1}{\rho} \frac{d}{d \rho} \psi(\rho)+ \\
& +\left(\eta-\frac{m^{2}}{\rho^{2}}-\alpha^{2} \rho^{2}-\beta^{4} \rho^{4}\right) \psi(\rho)=0,
\end{aligned}
$$

where

$$
\eta=\frac{2 m^{*}}{\hbar^{2}} E-k_{z}^{2} ; \quad \alpha^{2}=\frac{2 e A_{0} k_{z}}{\hbar} ; \quad \beta^{4}=\frac{e^{2} A_{0}^{2}}{\hbar^{2}}
$$

It is convenient to use the new variable in (6) $\xi=\alpha \rho^{2}$, and taking into account the asymptote for function $\psi(\rho)$ we present it in the form

$$
\psi(\rho)=\xi^{\frac{|m|}{2}} e^{-\frac{\xi}{2}} R(\xi),
$$

and, as result, we obtain the equation for $R(\xi)$

$$
\xi R^{\prime \prime}+(|m|+1-\xi) R^{\prime}+\left(a-\kappa \xi^{2}\right) R=0,
$$

where $a=\frac{\eta}{4 \alpha}-\frac{1}{2}(|m|+1) ; \kappa=\frac{\beta^{4}}{4 \alpha^{3}}$.

We should note that equation (8) differs from the commonly known case for uniform field by the term $\kappa \xi^{2}$ [4]. Let us find the solutions of equation (8). In this connection, we note here that for the uniform field $(\kappa=0)$ equation (8) has solutions in the form of degenerated hypergeometrical function $M(\xi)$ [5] and function $\psi(\rho)$ in this case is given by $\psi(\rho)=\rho^{|m|} \alpha^{\frac{|m|}{2}} e^{-\frac{\alpha^{2}}{2} \rho^{2}} M\left(-a, c, \alpha \rho^{2}\right)$,

where $c=|m|+1$.

We use the known method of presenting the function $R(\xi)$ in the form of

$$
R(\xi)=\sum_{j=0} A_{j} \frac{1}{j !} \xi^{j}
$$

in order to find the solutions of equation (8) when the coefficient $\kappa$ has a significant value.

For any values $a, c$ and $\kappa$ the existence of the irregular critical point in equation (8) at $\xi \rightarrow \infty$ does not guarantee convergence of series (10). Nevertheless, in this case such a procedure appears to be successful due to some restrictions for quantity $a$.

Making the standard calculations we obtain the recurrence relationship for the coefficients $A_{j}$

$$
A_{j+3}=\frac{1}{(j+3)(c+j+2)}\left\{A_{j+2}(j+2-a)+A_{j} \kappa\right\} .
$$

As can be seen from (10) and (11) the presence of $\kappa \xi^{2}$ in Eq. (8) is pronounced in the $A_{j}$ coefficient values, starting from $j=3$. As far as in the case of $\kappa=0$ the recurrence relationship (11) converges to the hypergeometric series, so it is possible to suggest that the first three coefficients in (10) have the following values

$$
A_{0}=1 ; \quad A_{1}=\frac{-a}{c} ; \quad A_{2}=\frac{(-a)(-a+1)}{2 c(c+1)} .
$$

Such a dependence between the coefficients gives the reason for consideration of series (10) as a sum of the three series, in each of which realized is the connection between neighbor coefficients. In turn, it causes the dependences between the triplets of coefficients, each of which is formed according to the power of $\kappa$. This is clearly manifested in the expression for an arbitrary coefficient $A$, which has the following form

$A_{j}=\frac{1}{j !(c)_{j}}\left\{(-a)_{j}+\sum_{s=1}^{i} \kappa^{s} \times\right.$

$\times \sum_{g=3}^{j} \prod_{l=1}^{i}(g+1-3 l)(g+2-3 l)(c+g-3 l)(c+g+1-3 l) \times$

$\left.\times \prod_{k=0}^{j-1}(-a+k)^{\prime}\right\}$

where $i$ is the number of triplets among the $j$-coefficients, and in the product $\prod_{k=0}^{j-1}(-a+k)^{\prime}, k$ does not take values 
J.M. Stakhira, R.J. Stakhira: The energy structure of free electrons for ...

$(g-3 l),(g+1-3 l),(g+2-3 l)$, also used $(a)_{j}=$ $=a(a+1)(a+2) \ldots(a+j-1)$.

Therefore, all the coefficients in series (10) have a specific expression and the $R(\rho)$ function (7) can be presented finally in an explicit form.

\section{Quantization of electron energy}

The relationships between the coefficients in series (10) somewhat complicate the procedure for satisfying the quantum mechanical conditions for the wave function $\psi(x, y, z)$. As follows from the properties of the coefficient system $A_{j}$, the transformation of series (10) to polynomial is possible only in the case of simultaneous equality to zero of all the coefficients of those triplets of neighbor coefficient, which cut off the series. In contrast to [4], this can not be reached by the only requirement of equality of $a(8)$ to positive integer, because the coefficient with summand without quantity $a$, exists for each triplet in expression (13).

In order to obtain the conditions of the truncation off for series (10) in respect to the quantity $a$, it is reasonable to present the expression for each coefficient $A_{j}$ in a form somewhat different (13), collecting adjacent in respect to the degree $k$ summands. As a result, each coefficient of the series can be presented as a sum of the products

$K(c, \kappa)\left|(-a+g)^{3}-(-a+g)+\kappa g(g+1)(c+g)(c+g-1)\right| \times$ $\times \prod_{k \neq g}^{j-1}(-a+k)=0$,

where $K(c, \kappa)$ is independent of $a$ and $g \geq 1$. To satisfy the equality of any triplet coefficients to zero in the most simple case, it is possible by requiring the equality to zero of all the summands (14) for each coefficient. It is possible only if the quantity $a$ equals to positive integer and, as a result of this condition, it will satisfy the cubic equation

$(-a+g)^{3}-(-a+g)+\kappa q=0$,

where $q=g(g+1)(c+g)(c+g+1)$.

We should note here that the integer real roots of equation (15) determine the permitted values of quantum numbers $g=n_{g}$ and $c=m_{g}$. These values are essentially limited by the quantity $\kappa$. The diagram of $n g$ values and the corresponding combination of quantum numbers that are responsible for equality (15) at different values of $\kappa=\beta^{4} / \alpha^{3}$ is shown in Fig. 1.

Hence, for the energy of electrons we have

$E=\frac{\hbar^{2} k^{2}}{2 m^{*}}+\frac{\hbar^{2} \alpha}{m^{*}}\left(2 N+m_{g}+1\right)$,

where the numbers $N, m_{g}$ satisfy equality (15). For the large ratio values $\kappa(\kappa>>0.04)$ the approximate value of energy has the form

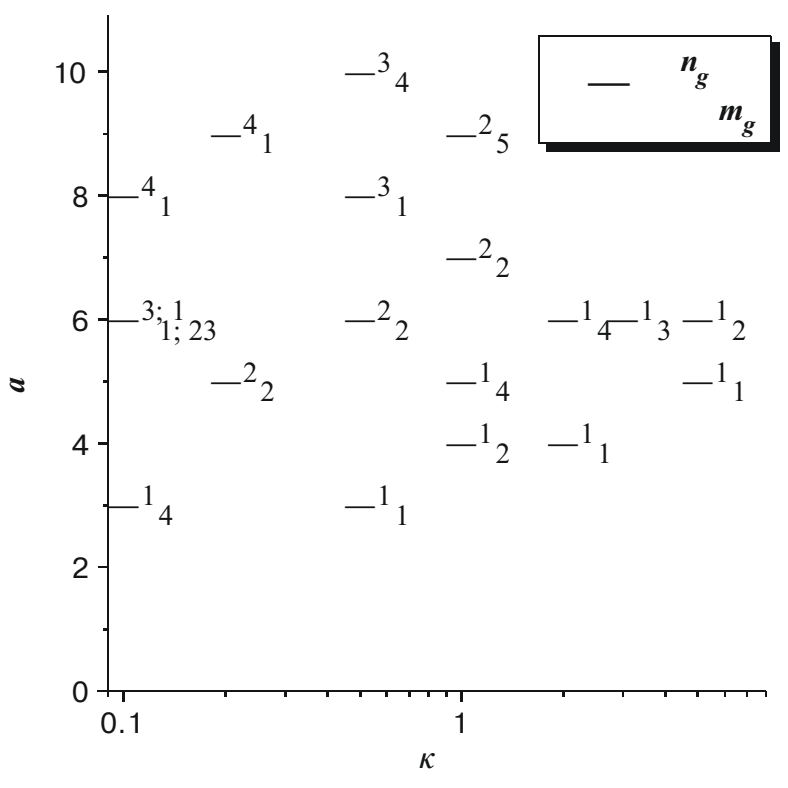

Fig. 1. Dependence of quantum numbers on the coefficients ratio for quadratic and biquadratic parts of the potential.

$E=\frac{\hbar^{2} k^{2}}{2 m^{*}}+\frac{\hbar^{2} \alpha}{m^{*}}\left(2 n_{g}+m_{g}+1\right)+\frac{2 \hbar^{2}}{m^{*}} \beta^{4 / 3} q^{1 / 3}$

where the $q$ is the product of permitted quantum numbers $m_{g}$ and $n_{g}$.

Let's note that the last term in (17) does not depend on the quantity $\alpha$ and is in qualitative agreement with the expression for the ground state energy of oscillator in considerable field, which was obtained by perturbation theory [6].

\section{Conclusion}

Now we would like to analyze some features of the obtained results. First of all, it should be marked that the occurrence of the biquadratic component in the potential causes essentialy influences the energetic structure of electrons. This influence is mainly pronounced in drastic restrictions for permitted quantum numbers.

In connection with this, there is a characteristic fact that in non-uniform field not only the spectrum of quantum numbers but their values also depend on the fraction of quadratic and biquadratic components. It is related also to the values of energy for the ground state that is formed by the combination of two quantum numbers for the considered fields. The values of these numbers show non-monotonic dependence on the ratio of quadratic and biquadratic components, although, as can be seen from Fig. 1, the energy of ground state has an explicit tendency to grow with increasing $\kappa$.

It should be noted that this is the most important result that can be interesting from the practical point of 


\section{J.M. Stakhira, R.J. Stakhira: The energy structure of free electrons for ...}

view and is connected with the density of energetic levels. In contrast to the uniform fields [4], the spectrum of permitted values of energy in potential of cylindrical symmetry is characterized by significant decrease in density, what is connected with the more rigid conditions for the permitted quantum numbers. According to this fact the energy gap between the levels in a ununiform field can be few times larger in comparison with the uniform field, and the explicit tendency to its growing with $\kappa$ increasing is observed.

For this reason, we pay attention to the experimental results $[7,8]$ that stimulated the interest to this study. As it was pointed out in these papers, the piezophotoconductivity spectrum as a component of conductivity is proportional to the product of light intensity and periodic mechanical pressure for certain layered semiconductors shows the oscillation dependence, and the period of such oscillations can reach $0.1 \mathrm{eV}$.

Finally, we should note that some part of conclusions of this work can pertain also to other physical processes described by means of the differential equation of the kind (4).

\section{References}

1. V.M. Tkachuk and S.I. Vakarchuk, Ground state of electron in the magnetic field of a straight current // J.Phys. A: Math.Gen. 34, pp. 653-662 (2001)

2. Baoling Wang, Shkange Yin, Guanghon Wang and Jijun Zhao, Structures and electronie properties of ultrathin titanium nanowires // J. Phys. Condens. Matter. 13, pp. 403-409 (2001)

3. M. Wilkinson, Critical properties of electron eidenstates in incommensurate system // Proc. R. Soc. Lond. A 391, pp. 305-350 (1984)

4. L.D. Landau, E.M. Livshits, Quantum mechanics. Nonrelativistic theory, Nauka, Moscow (1963)

5. M.A. Abramowitz, I.A.Stegun, Hypergeometrical function, Chap. 13 in Handbook of Mathematical Functions, Nauka, Mockow (1979)

6. I.O. Vakarchuk, Quantum mechanics, Lviv Ivan Franko state university, Lviv (1998)

7. J.M. Stakhira, Quantization of carriers of current in layered semiconductors // Phys. and Techn. of Semiconductors. 18 pp. 176-181, (1982)

8. J.M. Stakhira and R.J. Stakhira, Piezophotoconductive spectra in monopolar semiconductor // Journal of Physical Studies. 2, pp. 376-383 (1998). 